\title{
MULTIPRONGED QUANTITATIVE PROTEOMICS ANALYSIS OF PLASMODIUM VIVAX INDUCED ALTERATIONS IN HUMAN SERUM FOR IDENTIFICATION OF POSSIBLE BIOMARKERS FOR SEVERE VIVAX MALARIA
}

\section{Gajanand Singh Tanwar*, Priya Tanwar, Anuja Sapre, Heena Gahlot, Renu Agrawal; Department of Pediatric Medicine, S.P. Medical College, Bikaner}

\section{Background \& objectives}

The burden of malaria continues to worsen globally with a devastating impact on human health and corresponding impediment to economic improvement. Despite worldwide initiatives, emerging drug resistance in different species of Plasmodium and paucity of information about the exact underlying mechanism of the disease pathogenesis are creating challenges for the management and eradication of the disease. In addition, recent incidences of involvement of Plasmodium vivax in severe malaria suggest a drastic shift in the clinical paradigm for vivax malaria also. Investigation of the parasite induced alterations in host proteome and modulation of different vital physiological processes have great clinical relevance in the light of diagnosis and prognosis of non-severe vivax malaria (NSVM) and severe vivax malaria (SVM). Mechanisms that trigger transition from NSVM to SVM are obscure. Recently, proteomic studies have contributed substantially to our understanding of the clinical proteome of human malaria parasites, profiling humoral immune responses to Plasmodium vivax infection and the malaria parasite infection-induced changes in host erythrocyte membrane proteins. In 15 his context, this multidisciplinary study provides a comprehensive analysis of serum proteomics profile of NSVM and SVM to understand the itinerary during the ontogenetic advancement of the disease into severe forms and longitudinal analysis of the P.vivax infected children to identify surrogate markers of severity.

\section{Meterials and Methods}

This prospective cohort study was conducted on 126 children of P.vivax malaria admitted from January 2017 to December 2017. The species diagnosis was made with peripheral smear and rapid diagnostic test and confirmed with polymerase chain reaction analysis. Severe malaria was defined as per WHO guidelines. Serum samples from children with NSVM $(\mathrm{n}=30)$ and SVM ( $\mathrm{n}=30)$ were analysed in comparison to healthy controls $(\mathrm{HC})(\mathrm{n}=50)$ and dengue illness as febrile control (FC) ( $\mathrm{n}=45)$ by 2D-differential in gel electrophoresis (2D-DIGE) and isobaric tags for relative and absolute quantitation (iTRAQ)-based quantitative proteomics in combination with electrospray ionization quadrupole time-offlight (ESI-Q-TOF) and Q-Exactive mass spectrometry platforms. The results were validated by employing enzymelinked immunosorbent assay (ELISA).

\section{Results}

Comparative proteomics analysis of NSVM, SVM, HC and FC revealed evidences for the modulation of diverse physiological pathways including oxidative stress, cytoskeletal regulation, lipid metabolism and complement cascades in P.vivax malaria.
Proteins like complement C3, alpha-2-macroglobulin, complement C5, hemopexin, plasminogen, alpha-1antichymotrypsin, apolipoprotein A-IV, prothrombin, antithrombin-III, inter-alpha-trypsin inhibitor heavy chain H3, gelsolin \& apolipoprotein A-I showed similar trends of differential abundance in malaria patients (compared to healthy subjects) in both Q-TOF and Q-Exactive analyses. Interestingly, many serum proteins such as Superoxide dismutase, C-Reative Protein, Apolipoprotein E, Apolipoprotein A-1, Serum amyloid A, and Haptoglobin displayed significantly ( $p \leq .0001)$ different serum abundances in SVM compared to NSVM. Differential expression of these six serum proteins had correlation with the disease severity and therefore can be eventually used as powerful indicators for disease severity.

\section{Conclusion}

In summary, results obtained from this comprehensive proteomics analysis revealed activation of the oxidative stress and counteractive pathways, as well as elevated serum levels of cytoskeletal proteins as the possible clues contributing towards SVM. In this comprehensive multidisciplinary prospective study, 6 serum proteins were identified as potential predictive markers for P.vivax malaria severity. 